\title{
Interaction between Poly(vinyl alcohol) and Poly(ethylene glycol) in Water Studied by Viscosity and Density
}

\author{
Isamu INAMURA and Yuji JiNBO \\ Department of Chemistry, Faculty of Science, \\ Shimane University, Matsue 690, Japan
}

(Received November 28, 1990)

\begin{abstract}
KEY WORDS Viscosity/Density/Poly(vinyl alcohol)/Poly(ethylene glycol) / Water / Phase Separation / Additivity / Interaction /
\end{abstract}

One of the authors has investigated a liquid-liquid phase separation in a poly(vinyl alcohol) (PVA)-poly(ethylene glycol) (PEG)water system. ${ }^{1,2} \mathrm{We}$ are interested in an interaction between PVA and PEG in water in connection with the phase separation. To evaluate it, viscosity and density in the ternary system were measured over a range of polymer concentration up to high concentration, where a liquid-liquid phase separation sometimes occurred. We clarified the effects of the occurring of the phase separation on the concentration dependences of viscosity and density. To evaluate the interaction between PVA and PEG, we obtained the interaction parameter, $\Delta b_{23}$, by the method of Krigbaum and Wall. ${ }^{3}$ The specific viscosity and partial specific volume of polymer mixtures were also studied on the basis of additivity.

\section{EXPERIMENTAL}

\section{Materials}

Poly(vinyl alcohol) (PVA), PVA-105 and PVA-117 with $98.5 \mathrm{~mol} \%$ saponification, were kindly donated by Kuraray Co., Ltd. Poly(ethylene glycol) (PEG) manufactured by Kishida Chemical Co., Ltd., was used. PVA and PEG were purified and then PVA was fractionated by the method described previous- ly. ${ }^{1}$ The weight and number average molecular weights $\left(M_{w}\right.$ and $\left.M_{n}\right)$ of PVA and PEG samples were estimated by gel permeation chromatography (GPC). The ratio $M_{w} / M_{n}$ is from 1.10 to 1.57 . Thus, the molecular weight distributions of polymers used are moderately narrow. The characteristics of the polymer samples are given in Table I. PVA-1 sample was used for measurements to obtain the data in Figures 1, 2, 6, and 7, while PVA-2 sample used for measurements to get the data in Figures 3-5.

\section{Measurements of Viscosity and Density}

Ubbelohde and Ostwald viscometers were used to obtain viscosity, and a Lipkin-Davison pycnometer used to get density. The measurements of viscosity and density were performed at $30 \pm 0.01{ }^{\circ} \mathrm{C}$ over a range of polymer concentration up to $3-4 \mathrm{~g} \mathrm{dl}^{-1}$, where a liquidliquid phase separation sometimes occurred in the solution. The marks $\boldsymbol{\square}$ and $\boldsymbol{\Delta}$ in Figure 1 and the mark in Figures 2 and 6 represent the phase separation. The aqueous

Table I. Characteristics of the polymer samples

\begin{tabular}{lcc}
\hline Sample code & $M_{w} \times 10^{-4}$ & $M_{w} / M_{n}$ \\
\hline PVA-1 & 23.7 & 1.57 \\
PVA-2 & 3.94 & 1.37 \\
PEG & 2.10 & 1.10 \\
\hline
\end{tabular}


polymer solutions were filtered through a membrane filter (pore size $0.45 \mu \mathrm{m}$ ) to separate dust and undissolved gels. The solutions of polymer mixtures were prepared by mixing a PVA solution and a PEG solution. The weight ratio $\mathrm{PVA} / \mathrm{PEG}$ in the solutions were $1 / 3,1$ and 3 .

\section{Calculation of Specific Viscosity Based on Additivity}

The calculated curves of specific viscosity $\eta_{\mathrm{sp}}$ for polymer mixtures (in Figure 1) were based on additivity of specific viscosity with respect to the polymer composition. That is, the specific viscosity, $\eta_{\mathrm{sp}, \mathrm{m}}$ for a polymer mixture (weight fractions of PVA and PEG were $w_{2}$ and $w_{3}$, respectively; $\left.\left(w_{2}+w_{3}=1\right)\right)$ at polymer concentration $C_{\mathrm{m}}$ was calculated from the observed values of specific viscosities, $\eta_{\mathrm{sp}, \mathrm{PVA}}$ and $\eta_{\mathrm{sp}, \mathrm{PEG}}$ of single polymer solutions as follows;

$\eta_{\mathrm{sp}, \mathrm{m}} \quad\left(\right.$ at $\left.\quad C_{\mathrm{m}}\right)=\eta_{\mathrm{sp}, \mathrm{PVA}} \quad\left(\right.$ at $\left.\quad w_{2} C_{\mathrm{m}}\right)+\eta_{\mathrm{sp}, \mathrm{PEG}}$ (at $w_{3} C_{\mathrm{m}}$ )

The calculated curves in Figure 1 were drawn by plotting $\eta_{\mathrm{sp}, \mathrm{m}}$ against $C_{\mathrm{m}}$.

\section{RESULTS AND DISCUSSION}

\section{Viscosity}

Figures 1 and 2 shows the specific viscosity $\eta_{\text {sp }} v s$. polymer concentration $C$ and the reduced viscosity $\eta_{\mathrm{sp}} / C v s$. $C$, respectively, for PVA-PEG-water system. As is seen from these figures, the shapes of observed curves of $\eta_{\mathrm{sp}}$ and $\eta_{\mathrm{sp}} / C$ for polymer mixtures changed continuously in order of the weight ratio PVA/PEG.

The observed curves of $\eta_{\mathrm{sp}} v s . C$ plot for all polymer mixtures in Figure 1 positively deviated from the calculated ones at concentrations above $1.2 \mathrm{gdl}^{-1}$. Williamson and Wright ${ }^{4}$ described that the positive deviation of $\eta_{\mathrm{sp}}$ from additivity may be regarded as good compatibility of the polymer pair, while the negative deviation shows that compatibility is poor.

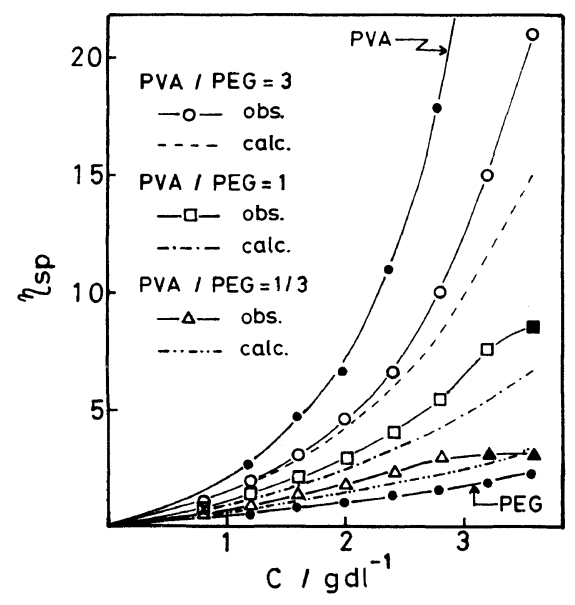

Figure 1. Specific viscosity vs. polymer concentration for PVA-PEG-water system. The marks $\boldsymbol{\square}$ and $\boldsymbol{\Delta}$ indicate that a liquid-liquid phase separation occurred in the solution.

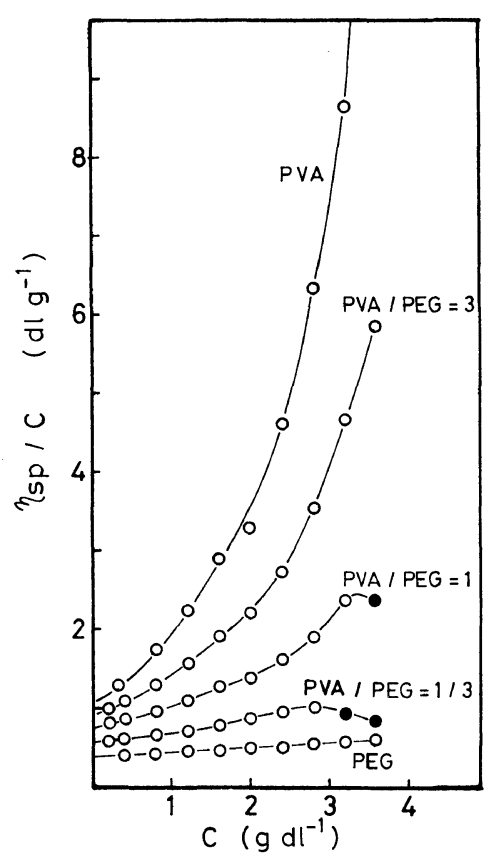

Figure 2. Reduced viscosity vs. polymer concentration for PVA-PEG-water system. O, liquid-liquid phase separation.

Quadrat, ${ }^{5}$ however, claimed that the additivity of $\eta_{\mathrm{sp}}$ cannot become the property to judge a compatibility of polymers. Therefore, the 
origin of the positive deviation of $\eta_{\mathrm{sp}}$ from additivity, observed in this work, for PVAPEG-water system should be elucidated in future. However, it can be inferred that an increase in the number of entanglements of polymer chains by mixing two kinds of polymers may be responsible for the positive deviation.

As seen from Figures 1 and 2, on occurring of a liquid-liquid phase separation at high concentrations, both $\eta_{\mathrm{sp}}$ and $\eta_{\mathrm{sp}} / C$ decreased, and $\eta_{\mathrm{sp}}$ negatively deviated from additivity. We have ascertained from the phase diagram of PVA-PEG-water system ${ }^{1}$ that a polymer mixture solution separates into a concentrated PVA-rich and a dilute PEG-rich phase. Therefore, the existence of dilute PEG-rich phase is considered to cause the decrease in $\eta_{\mathrm{sp}}$ and $\eta_{\mathrm{sp}} / C$.

Figure 3 shows $\eta_{\mathrm{sp}} / C$ vs. $C$ for dilute PVA-PEG-water system. All Huggins plots gave a straight line. The intrinsic viscosity $[\eta]$ and Huggins constant $k^{\prime}$ were evaluated from the intercept and slope of the plots, respectively.

Figure 4 shows the polymer composition dependences of $[\eta]$ and $k^{\prime}$. A plot of $[\eta] v s$. PVA content was found to be linear. Such linear relationship was also observed for [ $\eta]$ obtained from Figure 2 (data not shown). This suggests the additivity of $[\eta]$ with respect to polymer composition. Though additivity of $[\eta]$ is generally observed in polymer-polymersolvent systems, ${ }^{6}$ it is noted that it is also observed in PVA-PEG-water system showing a liquid-liquid phase separation at high concentration. The Huggins constant $k^{\prime}$ increased from 0.182 to 0.643 with increasing PVA content. It is well known that $k^{\prime}$ reflects the solute-solute interactions. ${ }^{7}$

Using the Huggins plots in Figure 3, the polymer 2(PVA)-polymer 3(PEG) interaction parameter, $\Delta b_{23}$, was calculated by the method of Krigbaum and Wall. ${ }^{3}$ Figure 5 shows $\Delta b_{23}$ against PVA content. The $\Delta b_{23}$ values are negative, though the absolute values are small.

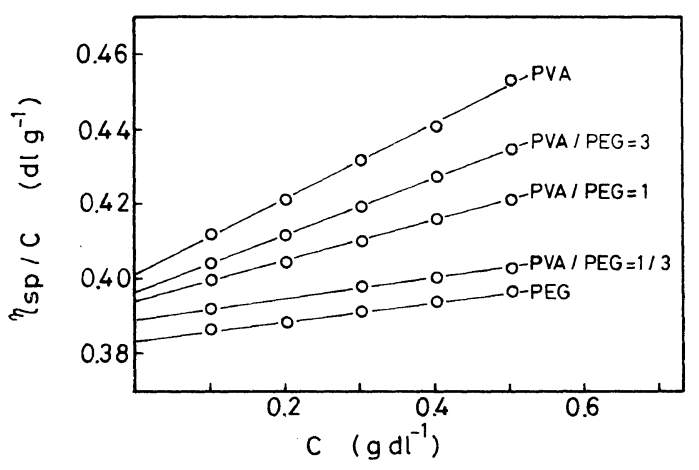

Figure 3. Reduced viscosity $v s$. polymer concentration for dilute PVA-PEG-water system.

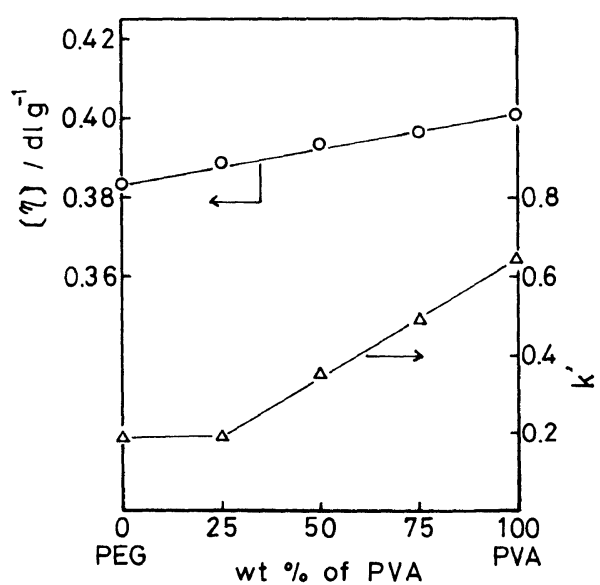

Figure 4. Polymer composition dependence of intrinsic viscosity and Huggins constant obtained from Figure 3.

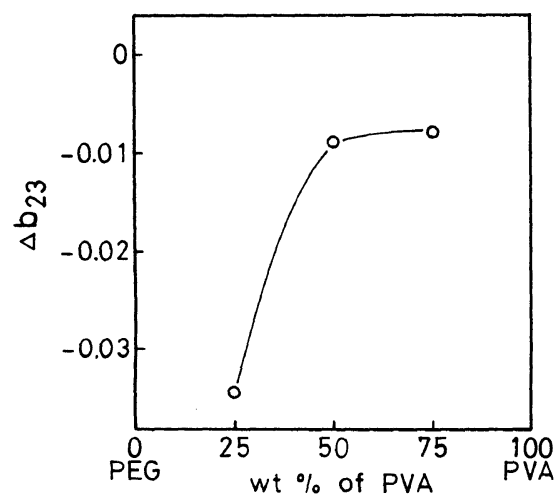

Figure 5. Polymer composition dependence of interaction parameter between PVA and PEG. 
Further it is noted that $\Delta b_{23}$ decreased with increasing PEG content and reached to the minimum value at $25 \mathrm{wt} \%$ of PVA. According to Krigbaum and $\mathrm{Wall},{ }^{3}$ positive $\Delta b_{23}$ values indicate attractive interations and compatibility, whereas negative values do repulsive interactions and incompatibility. Therefore, it can be inferred that a weak repulsive interaction exists between PVA and PEG in PVA-PEG-water system. Recently, Suto et al. ${ }^{8}$ investigated the viscometric hevavior of aqueous solutions of polymer mixtures involving a liquid crystalline polymer, and reported that there exist repulsive interactions between hydroxypropyl cellulose (liquid crystalline polymer) and hydroxyethyl cellulose.

\section{Density}

Figure 6 shows the density $\rho$ vs. polymer concentration for PVA-PEG-water system. The density increased linearly with increasing concentration in all cases. It is very interesting that the linear relationship is retained even at high concentrations where a liquid-liquid phase separation occurred. This result means that the overall density of a polymer mixture solution is not affected by the separation of solution into two phases with different densities. We will examine in the near future whether this behavior of density can be observed or not in other polymer-polymersolvent systems.

The partial specific volumes $\bar{v}$ were calculated from the slopes of the straight lines of density in Figure 6, and these values are also noted in this figure. Figure 7 shows $\bar{v}$ vs. PVA content. The $\bar{v}$ values upward deviated from the straight lines which corresponded to additivity. It is noted that the $\bar{v}$ value of the polymer mixture with PVA content equal to $50 \mathrm{wt} \%$ was extraordinarily high, being higher than that of PEG. Such a positive deviation of $\bar{v}$ seems to imply that there exists a repulsive interaction between PVA and PEG. This is in good agreement with the suggestion derived from the interaction parameter $\Delta b_{23}$. The difference in

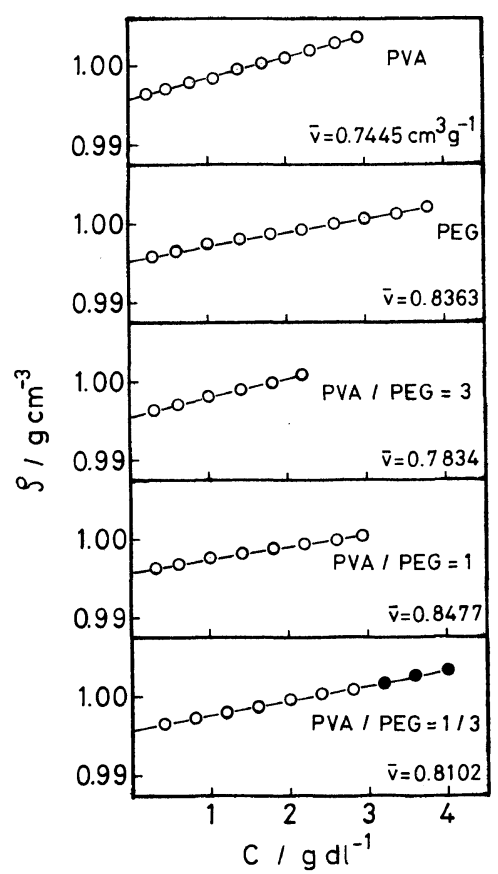

Figure 6. Density vs. polymer concentration for PVAPEG-water system. $\mathbf{0}$, liquid-liquid phase separation.

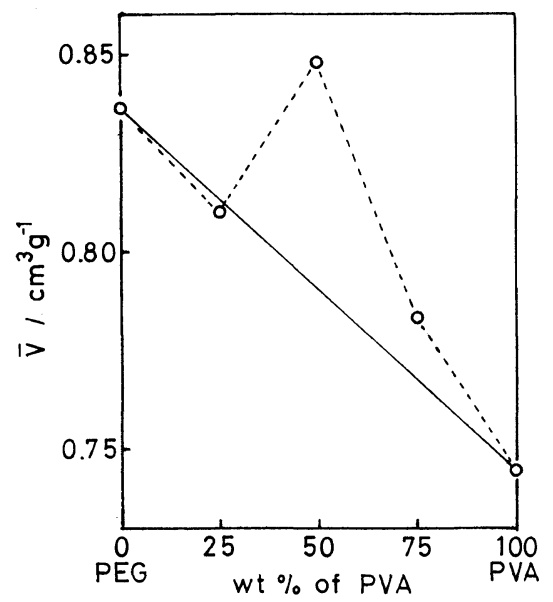

Figure 7. Polymer composition dependence of partial specific volume.

PVA content giving the maximum $\bar{v}$ value (50wt $\%$ PVA) and the minimum $\Delta b_{23}$ value (25 wt \% PVA) may be partly due to the difference in $M_{w}$ of PVA samples used. 


\section{CONCLUSION}

The occurring of a liquid-liquid phase separation decreased the slopes of $\eta_{\mathrm{sp}}$ vs. $C$ and $\eta_{s p} / C$ vs. $C$ plot. However, it did not change the slope of density $\rho$ vs. $C$ plot. Both $\eta_{\text {sp }}$ and $\bar{v}$ values positively deviated from additivity, whereas additivity of $[\eta]$ was observed. The interaction parameter $\Delta b_{23}$ was found to be negative, which suggests that a weak repulsive interaction exists between PVA and PEG.

\section{REFERENCES}

1. I. Inamura, K. Toki, T. Tamae, and T. Araki, Polym. J., 16, 657 (1984)

2. I. Inamura, Polym. J., 18, 269 (1986).

3. W. R. Krigbaum and F. T. Wall, J. Polym. Sci., 5, 505 (1950).

4. G. R. Williamson and B. Wright, J. Polym. Sci., A, 3, 3885 (1965).

5. O. Quadrat, Makromol. Chem., Rapid Commun., 1, 651 (1980).

6. W. Philippoff, Berichte d. D. Chem. Gesellshaft, 70, 827 (1937).

7. L. H. Cragg and C. C. Bigelow, J. Polym. Sci., 16, 177 (1955).

8. S. Suto, M. Oshima, O. Takatsu, and M. Karasawa, Kobunshi Ronbunshu, 43, 237 (1986). 